Research Paper

\title{
On the General Solutions of a Nonlinear Pseudo-Oscillator Equation and Related Quadratic Liénard Systems
}

\author{
ELÉMAWUSSI APÉDO DOUTETIEN ${ }^{1}$, AYÉNA RÉGIS YEHOSSOU ${ }^{1}$, PRAVANJAN MALLICK ${ }^{2}$, \\ BISWANATH RATH ${ }^{2}$ and MARC DELPHIN MONSIA ${ }^{1, *}$ \\ ${ }^{1}$ Department of Physics, University of Abomey-Calavi, Abomey-Calavi, 01.BP.526, Cotonou, Bénin \\ ${ }^{2}$ Department of Physics, North Orissa University, Takatpur, Baripada 757 003, Odisha, India
}

(Received on 27 August 2019; Accepted on 29 September 2019)

\begin{abstract}
We compute explicitly the exact and general solutions of the so-called pseudo-oscillator equation $x \ddot{x}+1=0$, and of its related quadratic Liénard type equations in a straightforward and direct method. This is achieved using the generalized Sundman transformation theory introduced recently in the literature by some authors of this work.
\end{abstract}

Keywords: Nonlinear Pseudo-Oscillator Equation; Nonlocal Transformation; Exact and General Solution

\section{Introduction}

One of the most investigated truly nonlinear oscillator equation is the singular equation

$$
\ddot{x}+\frac{1}{x}=0
$$

It is interesting to notice that equation (1) is of physical importance (Acton and Squire, 1985; Nayfeh and Mook, 1979) and is widely studied in the literature using different exact or approximate methods to compute periodic solutions (Mickens, 2007; Beléndez et al., 2008, 2008a; Mirzabeigy et al., 2012; Xu, 2011). By analyzing the so-called pseudo- oscillator equation (1), the authors in (Gadella and Lara, 2014) found that such equation has no smooth periodic solutions (Van Gorder, 2015), but, instead, admits two exact and general non-periodic solutions. Indeed, they have considered the equation (1) and rewrote it as a planar autonomous dynamical system for which, they found the Hamiltonian and the associated vector field. By analysis of the components of this vector, they observed that the phase plane trajectories presented by the system eliminate the existence of periodic solution of (1). They have deduced that the solution of (1) $x=\varphi(t)$ is invertible and its inverse function is given by $\mathrm{t}=\varphi^{-1}(x)=f(x)$. Using the change of variable $t=f(x)$, they have rewritten equation (1) in a form of an equation which was solved by quadrature. They have inverted the solution found and obtained the general solution of (1) given by

$$
x(t)=\sqrt{\frac{2}{\pi}} c_{1} \exp \left\{-\left(\operatorname{erf}^{-1}\left(\mp \frac{t-c_{2}}{c_{1}}\right)\right)^{2}\right\}
$$

To find the second solution, they claimed to turn the sign of a positive constant of integration into a negative sign. However, the method used by these authors (Gadella and Lara, 2014) does not allow the explicit and direct calculation of the two general solutions. Hence, one can see that only one general solution (1-a) has been exhibited explicitly by the authors (Gadella and Lara, 2014). Moreover, in their comment (Mancas and Rosu, 2016), the authors have only succeeded to exhibit the single general solution (1-a).

The goal of this paper is to show in a straightforward manner that the two exact and general solutions of equation (1) highlighted in (Gadella and Lara, 2014) can be explicitly computed and to investigate explicitly the related quadratic Liénard type

*Authorfor Correspondence: E-mail: monsiadelphin@yahoo.fr; biswanathrath10@gmail.com 
equations. To do so, equation (1) is shown to be a nonlocal transformation of the well-known linear harmonic oscillator equation (section 2), so that the general solutions of (1) may be easily computed and discussed (section 3). Finally, the related quadratic Liénard type equations are investigated (section 4) and a conclusion is carried out for the work.

\section{Nonlocal Transformation of Equations}

Consider the linear harmonic oscillator equation with forcing constant term.

$$
y^{\prime \prime}(\tau)+a^{2} y(\tau)=c
$$

where, prime denotes differentiation with respect to $\tau, a$ and $c$ are arbitrary parameters, and the nonlocal transformation (Koudahoun et al., 2019)

$$
\begin{aligned}
& y^{m}(\tau)=\int g^{l}(x) d x \\
& d \tau=g^{l}(x)\left[\int g^{l}(x) d x\right]^{\left(\frac{1}{m}-1\right)} d t
\end{aligned}
$$

$l$ and $m$ are arbitrary parameters. Therefore, applying the nonlocal transformation (3) to (2) leads to a general class of equations (Koudahoun et al., 2019).

$$
\begin{aligned}
& \ddot{x}+a^{2} m g^{l}(x)\left[\int g^{l}(x) d x\right]^{\left(\frac{2}{m}-1\right)} \\
& -m c g^{l}(x)\left[\int g^{l}(x) d x\right]^{\left(\frac{1}{m}-1\right)}=0
\end{aligned}
$$

In this way, the following lemma may be proved.

\section{Lemma}

If $g(x)=x^{2}, m=2, c=0, l=-\frac{1}{2}$, and $2 a^{2}=1$, then equation (4) transforms into equation (1).

\section{Proof}

For $m=2, c=0,(4)$ becomes

$$
\ddot{x}+2 a^{2} g^{l}(x)=0
$$

which is

$$
\ddot{x}+\frac{2 a^{2}}{x}=0
$$

if, $g(x)=x^{2}$, and $l=-\frac{1}{2}$.

Finally, setting $2 a^{2}=1$, in (6) leads to the desired equation (1).

Now, we may compute the two exact and general solutions to equation (1).

\section{Exact and General Solutions to Equation (1)}

We, first, investigate in the following, the exact and explicit general solutions of equation (6) such that one may deduce the exact and general solutions of equation (1).

Substituting the values attributed previously to $m, 1$ and the expression of $g(x)$ in (3) yields

$$
y^{2}(\tau)=\int \frac{1}{x} d x, d \tau=\frac{1}{x}\left[\int \frac{1}{x} d x\right]^{\frac{-1}{2}} d t
$$

such that the general solutions to (6) is

$$
x(t)=\varepsilon \exp \left(y^{2}(\tau)\right)
$$

with, $\varepsilon=1$ if $x>0$, or $\varepsilon=-1$, if $x<0$. The solution of (2) for $c=0$, may be written as

$$
y(\tau)=A_{0} \sin (a \tau+\alpha)
$$

where, $A_{0}$ and $\alpha$ are arbitrary constants and $t$ satisfies

$$
\int y e^{y^{2}} d \tau=\varepsilon(t+k)
$$

that is

$$
\int A_{0} \sin (a \tau+\alpha) e^{A_{0}^{2} \sin ^{2}(a \tau+\alpha)} d \tau=\varepsilon(t+k)
$$

and $k$ is an integration constant.

Let us evaluate the left hand side of (11) by setting it as $J$.

Imposing $\theta=a \tau+\alpha$, yields

$$
a J=\int A_{0} \sin \theta e^{A_{0}^{2} \sin ^{2} \theta} d \theta
$$


Let

$$
u=A_{0} \cos \theta
$$

then

$$
d \theta=\frac{-d u}{A_{0} \sin \theta}
$$

and

$$
\sin ^{2} \theta=1-\frac{u^{2}}{A_{0}^{2}}
$$

Therefore

$$
-a J=e^{A_{0}^{2}} \int e^{-u^{2}} d u
$$

Knowing (Abramowitz and Stegun, 1972)

$$
\operatorname{erf}(z)=\frac{2}{\sqrt{\pi}} \int_{0}^{z} e^{-q^{2}} d q
$$

then

$$
J=\frac{-\sqrt{\pi}}{2 a} e^{A_{0}^{2}} \operatorname{erf}(u)
$$

Replacing (18) in (11) yields

$$
\operatorname{erf}(u)=\mp \frac{2 a}{\sqrt{\pi}} e^{-A_{0}^{2}}(t+k)
$$

from which

$$
u=\operatorname{erf}^{-1}\left[\mp \frac{2 a}{\sqrt{\pi}} e^{-A_{0}^{2}(t+k)}\right]
$$

Substituting (20) in (15) leads to

$$
A_{0}^{2} \sin ^{2} \theta=A_{0}^{2}-\left\{\operatorname{erf}^{-1}\left[\mp \frac{2 a}{\sqrt{\pi}} e^{\left.-A_{0}^{2}(t+k)\right]}\right\}^{2}\right.
$$

Thus, one may rewrite the solution (8) in the form

$$
x(t)=\varepsilon e^{A_{0}^{2}} \exp \left\{-\left[\operatorname{erf}^{-1}\left[\mp \frac{2 a}{\sqrt{\pi}} e^{\left.\left.-A_{0}^{2}(t+k)\right]\right]^{2}}\right\}\right. \text { (2) }\right.
$$

where, $\varepsilon= \pm 1$.

In this situation, the following theorem is proved for $a=\frac{\sqrt{2}}{2}$.

\section{Theorem}

If, $a=\frac{\sqrt{2}}{2}$, then the exact and general solutions of equation (6) are

$$
x(t)= \pm \sqrt{\frac{2}{\pi}} c_{1} \exp \left\{-\left[\operatorname{erf}^{-1}\left[\mp \frac{\left(t-c_{2}\right)}{c_{1}}\right]\right]^{2}\right\}
$$

where $c_{1}=\sqrt{\frac{\pi}{2}} e^{A_{0}^{2}}$ and $c_{2}=-k$.

Equation (23) is the desired exact and general solutions of equation (1). The positive value of (23) corresponds to the solution found in (Gadella and Lara, 2014). The theory developed in this work to compute the solutions of equation (1) doesn't lead to a periodic solution. Moreover, the relation between the harmonic oscillator equation and equation (1) eliminates any possibility of existence of the smooth periodic solution of equation (1). That being so, we may investigate the class of quadratic Liénard type equations related to (1).

\section{General Class of Quadratic Liénard Type Equations}

This section is devoted to investigate the general class of quadratic Liénard type equations (Kamke, 1977)

$$
\lambda u \ddot{u}+b \dot{u}^{2}+\mu u^{l}=0
$$

For, $l=0, b=0$ and $\frac{\mu}{\lambda}=1$, with $\lambda \neq 0$, equation (24) becomes identical to the pseudo-oscillator equation (1). The problem is now to ask whether equation (24) may include (1) as a special case and to be solved explicitly in terms of (23). It seems that such a problem has not been investigated previously in the literature. The interest is to find a new class of solutions of quadratic Liénard type equations. 
Now, let $\frac{b}{\lambda}=-(q+1), \frac{\mu}{\lambda}=-\frac{1}{q}, l=2 q+2$ and $q \neq 0$. Then (24) reduces to

$$
\ddot{u}-(q+1) \frac{\dot{u}^{2}}{u}-\frac{1}{q} u^{2 q+1}=0
$$

Hence, one deduces easily the following corollary from the theorem in above.

\section{Corrolary}

If the change of variable

$$
u=x^{\frac{-1}{q}}, x \neq 0
$$

holds, then equation (1) is mapped into (25).

\section{Proof}

By using the change of variable (26), one may obtain

$$
\frac{d x}{d t}=-q u^{-q-1} \frac{d u}{d t}
$$

and

$$
\frac{d^{2} x}{d t^{2}}=-q\left\{-(q+1) u^{-(q+1)-1}\left(\frac{d u}{d t}\right)^{2}+u^{-(q+1)} \frac{d^{2} u}{d t^{2}}\right\}
$$

\section{References}

Acton J R and Squire P T (1985) Solving Equations with Physical understanding. Techno House USA

Nayfeh A H and Mook D T (1979) Nonlinear oscillations. John Willey \& Sons

Mickens R E (2007) Harmonic balance and iteration calculations of periodic solutions to $y^{\prime \prime}+y^{-1}=0$ J Sound Vib 306968 72

Beléndez A, Méndez D I, Beléndez T, Hernàndez A and Alvarez M L (2008) Harmonic balance approaches to the nonlinear oscillators in which the restoring force is inversely proportional to the dependent variable $J$ Sound Vib $\mathbf{3 1 4}$ 775-82

Beléndez A, Gimeno E, Fernàndez E, Méndez D I and Alvarez M L (2008) Accurate approximate solution to nonlinear
Substituting (28) into (1), and using (26), yields immediately (25). Therefore, the corollary is proved. In this perspective, the exact and general solutions to (25) become

$$
u(t)=\left\{ \pm \sqrt{\frac{2}{\pi}} c_{1} \exp \left\{-\left[\operatorname{erf}^{-1}\left[\mp \frac{\left(t-c_{2}\right)}{c_{1}}\right]\right]^{2}\right\}\right\}^{\frac{-1}{q}}
$$

As expected, if $q=-1$, then (25) reduces to (1) and (29) coincides with (23).

\section{Conclusion}

Many authors have investigated the so-called pseudooscillator equation with different methods. In this work, the integrability of this equation is investigated using equation transformation theory. It has been possible to compute explicitly and exactly its exact and general solutions. It has been also possible to investigate a general class of quadratic Liénard type equations related to such a pseudo-oscillator equation. So, using a simple appropriate variable change, it is shown the existence of a new class of solutions for quadratic Liénard type equations.

oscillators in which the restoring force is inversely proportional to the dependent variable Phys Scr 77065004

Mirzabeigy A, Kalami-Yazdi M and Yildirim A (2012) Analytical approximations for a conservative nonlinear singular oscillator in plasma physics J Egyptian Math Soc 20163 6

Xu L (2011) A Hamiltonian approach for a plasma physics problem J Comp Math Appl 61 1909-1911

Gadella M and Lara L P (2014) On the solutions of a nonlinear 'pseudo'-oscillator equation Phys Scr 89105205

Van Gorder R (2015) continuous periodic of a nonlinear pseudooscillator equation in which the restoring force is inversely proportional to the dependent variable Phys Scr 90085208

Mancas S C and Rosu H C (2016) Existence of periodic orbits in nonlinear oscillators of Emden-Fowler form Phys Lett A 
$380 \quad 422-428$

Mickens R E (2007) Harmonic balance and iteration calculations of periodic solutions to J Sundy Vib 306 968-972

Koudahoun L H, Kpomahou Y J F, Akande J, Adjaï D K K and Monsia M D (2019) Integrability analysis of a generalized Truly nonlinear oscillator equation viXra.org/906.0212v2. pdf
Abramowitz M and Stegun I (1972) Handbook of Mathematical Functions with Formulas Graphs and Mathematical. Table New York Dover

Kamke E (1977) Differential gleichungenlösungs method en und lösungen. Springer Fachmedien Wiesbaden GMBH $10^{\text {th }}$ edition. 